\title{
Evaluation of Magnetic Resonance Imaging Findings and Short-Term Outcome in Brain Metastatic Tumors after CyberKnife Treatment
}

\author{
Jiashou Hu1, Hongzi Tian', Na Guo², Di Wang1, Jinfeng Sun² \\ ${ }^{1}$ Medical Imaging Department, The People's Liberation Army 107th Hospital, Yantai, China \\ ${ }^{2}$ The Whole Army Tumor Therapy Center, The People's Liberation Army 107th Hospital, Yantai, China \\ Email: jiashouhu@sina.com, 195468623@qq.com
}

Received 22 January 2016; accepted 26 February 2016; published 29 February 2016

Copyright (C) 2016 by authors and Scientific Research Publishing Inc.

This work is licensed under the Creative Commons Attribution International License (CC BY).

http://creativecommons.org/licenses/by/4.0/

(c) (i) Open Access

\begin{abstract}
Objective: To evaluate the treatments' outcomes in brain metastatic tumors after CyberKnife treatment according to magnetic resonance imaging (MRI) findings and improvement of symptoms. Methods: A retrospective analysis of CyberKnife treatment; 63 cases of patients with brain metastases; the use of CyberKnife treatment; short-term outcome evaluation after treatment and the MRI findings and measured before treatment and underwent diffusion-weighted imaging MRI scan of apparent diffusion coefficient (ADC) values. Results: 3 months after CyberKnife treatment and effectiveness were $82.5 \%$ and $96.8 \%$ respectively; 6 months and one year survival rates were $82.5 \%$ and $55.6 \%$ respectively; the median survival time was 16 months. MRI of 52 patients (67 lesions) ADC values after treatment increased to some extent than before treatment. There are 38 lesions volume to shrink or disappear, no enhancement or slight enhancement in the lesion, no edema zone; 27 lesions does not change in volume, no edema (18 lesions significantly weakened the degree of enhancement; 6 lesions showed no obvious change enhancement; 3 lesions showed ring enhancement, internal cystic); 2 lesions volume were larger, heterogeneous enhancement, peripheral edema. Conclusion: CyberKnife is an effective method for treating brain metastatic tumor. MRI can accurately evaluate tumor lesions after treatment.
\end{abstract}

\section{Keywords}

Brain Metastases, Radiation Therapy, CyberKnife 


\section{Introduction}

Extracranial malignant brain metastases are involved in the brain, skull and meninges, brain metastasis mostly in the lower part of the cerebral cortex and cortex, followed by the cerebellum, saddle area, cerebellopontine angle and brainstem.

Meningeal metastasis occurs on the inside of dura mater and pia mater. Common primary tumors of brain metastases include lung cancer, breast cancer, digestive tract, urinary tract and uterine ovarian tumor, thyroid, malignant melanoma, etc. Up to their primary tumor histological type is adenocarcinoma, followed by squamous cell carcinoma, papillary carcinoma and melanoma. More than $70 \%$ of brain metastases associated with neurological aspects of clinical signs and symptoms, followed by poor positioning and mental disorders and other symptoms. Treatment of brain metastases includes hormone therapy, whole brain radiation therapy, surgery and chemotherapy. At present, the radiation therapy plays an important role in the treatment of brain metastases tumor. The CyberKnife has as many as 1200 beams, and through the tumor from various angles, can increase the exposure dose of tumor site and minimize the normal tissue dose of illuminated, while increasing the tumor dose rate effectively reduces the complications of radiotherapy. CyberKnife is a new and sustainable use of image-guided robotics technology. Mobile features correspond exactly to the position of the patient radiation delivery system. CyberKnife's 6D skull tracking technology can take advantage of features in the healing process of bone sustained treatment of intracranial target tracking and automatically correcting even the most subtle movement and twisting will not miss. Now in our hospital 63 patients with brain metastases were treated with CyberKnife, and the results will now report as follows.

\section{Materials and Methods}

\subsection{General Information}

Select from November 2010 to December 2012 were treated brain CyberKnife treatment 63 cases of metastases patients, including 41 males and 22 females, aged 31 to 82 years, with an average age of 58 years; primary foci for lung cancer 43 cases; 11 cases of gastrointestinal cancer; breast cancer seven cases; 2 cases of lymphoma. Before treatment, KPS score $<70$ points 11 cases; $\geq 70$ points 52 cases. All brain metastases were confirmed by CT and MRI, in which single lesion in 48 cases; two lesions in six cases; three or more lesions in nine cases, a total of 98 brain metastases, tumor diameter of $0.8-8.8 \mathrm{~cm}$, the average diameter of $3.1 \mathrm{~cm} .52$ patients $(67 \mathrm{le}-$ sions) before treatment and MRI scan width San weighted imaging and measuring the apparent diffusion coefficient (ADC) values of the tumor region underwent treatment. Among them 53 cases of patients with tumor size, shape, location different, showing a headache, dizziness, nausea, vomiting, limb disorders, language disorders, memory losses and vision losses, such as different symptoms, clinical symptoms and the remaining ten patients were not obvious.

\subsection{Treatment Methods}

Patients with supine position, using hot mold fixed, cranial CT, MRI scan, CT scan slice thickness $1.25 \mathrm{~mm}$, MRI slice thickness $2.5 \mathrm{~mm}$, axial uninterrupted scan, determine the size of the lesion, shape and position of the transmission to the radio wave knife workstation to CT image-based, the CT image and MRI image fusion. Sketched by the physician in the treatment planning system target, then make plans by the physical division, and finally approved by a physician. Tumor volume of 15 - $35 \mathrm{~Gy}$, divided into 1 to 5 times with $70 \%$ to $80 \%$ isodose curve more than $95 \%$ of tumor volume. Including 12 patients underwent whole brain radiation therapy, 35 patients with chemotherapy treatment. This clinical trial was approved by the Institutional Ethics Committee and all the patients provided written informed consent.

\subsection{Efficacy Evaluation}

1) Symptoms improve evaluation standard: 1 week after treatment for clinical evaluation. Complete remission: No clinical positive performance after treatment; Partial remission: After treatment, the clinical symptoms are mild; Stable: No obvious clinical improvement after treatment; Increase: Clinical symptoms get worse after treatment. To (complete remission and remission) computing efficiency improvement of symptoms.

2) Recent radiological lesions Response Evaluation Criteria: Radiotherapy after 1 month review head CT or 
MRI, According to the WHO solid tumor treatment evaluation standard(RECIST) [1]: complete remission (CR), partial response (PR), stable (SD) and progression (PD), $(\mathrm{CR}+\mathrm{PR})$ called treatment improved, $(\mathrm{CR}+\mathrm{PR}+\mathrm{SD})$ called the treatment is effective.

\section{Results}

1) Short term effect: After one week of CyberKnife treatment, the clinical symptoms resolved 46 cases, 14 cases of partial remission, stable three cases, symptoms improved efficiency of $95.2 \%$. CyberKnife treatment of three months efficacy evaluation, CR 33 cases, PR 19 cases, SD 9 cases, PD 2 cases, treatment improved 82.5\% effective rate of $96.8 \%$. MRI was performed in 52 patients (67 lesions) were lower than before treatment ADC values corresponding contralateral normal tissue after treatment, review of ADC values higher than before treatment to varying degrees. MRI was performed in 67 lesions, 38 lesions volume to shrink or disappear, no enhancement or slight enhancement of lesions, no significant edema zone surrounding lesions; 27 lesions had no obvious change in the surrounding volume, no significant edema area, of which 18 lesions degree of enhancement significantly weakened, no obvious change in 6 lesions degree of enhancement, 3 lesions showed ring enhancement, internal cystic lesions; 2 volumes are increased, which showed heterogeneous enhancement, peripheral edema zone.

2) Survival and follow-up: Follow-up to January 2014, six-month survival rate was $82.5 \%$ (52 cases); 1 -year survival rate was $55.6 \%$ ( 35 cases); the median survival time was 16 months.

\section{Discussion}

In recent years, with the improvement of radiation therapy technology, whole brain radiation therapy has become a routine treatment for brain metastases. Whole Brain radiation therapy can not only improve brain metastases had a median survival time from 1 to 2 months symptomatic treatment alone increased to four to six months, but also can improve the quality of life of patients [2]. Surgery alone in patients with solitary brain metastases, the median survival time was 6.5 to 11.5 months, surgery plus whole brain radiation therapy can extend survival time to 10 to 16 months, but due to the patient's age, physical condition, tumor growth area and the number of tumor factors, surgical treatment is limited to some extent, cannot ensure complete resection of the tumor lesion Asia, leading patients may relapse within a period of time. Some patients will appear serious complications after tumor resection; reduce the quality of life, so the majority of patients do not accept surgical treatment.

Gamma knife for the treatment of brain metastases tumor local control is $81 \%-100 \%$, but gamma knife treatment of patients with brain metastases need to install a fixed frame of the head, to increase the patient's pain and sufferings, and only a single treatment [3] [4]. CyberKnife treatment is a new treatment for stereotactic radiotherapy, without a fixed time frame treatment of brain metastases, image-guided 6D skull tracking technology can take advantage of skeletal features in the course of treatment to keep track of intracranial target and automatically correct treatment, reducing the suffering of patients. In addition, the CyberKnife has as many as 1200 beams, can through the tumor from various angles, increase the exposure dose of tumor site and minimize the normal tissue dose of illuminated, while increase the tumor dose rate effectively reduce the complications of radiotherapy. The number of treatment used by CyberKnife according to the physical condition of patients and acceptable radiotherapy dose adjustments ( $1-5$ times), so as to realize the individualized treatment of patients. Further, since the reflected wave is a non-radioactive knife eccentric irradiation, the irradiation of each node may have more than two tumor irradiation, thus irradiating a treatment plan can be more tumors.

Young et al. [5] compared the $\gamma$ knife and CyberKnife treatment of brain metastases efficacy, $\gamma$ knife treatment of a single dose of $14-20 \mathrm{~Gy}, 50 \%$ isodose lines surrounding the tumor volume, tumor control rate was 96.7\%; while CyberKnife 14 - 30 Gy doses of 1 to 3 times, isodose curve $95 \%$ of the tumor volume, tumor control rate was $97.8 \%$. Nishida et al. [6] reported the results of a study of 71 patients, the treatment of tumor margins mean dose of $20.2 \mathrm{~Gy} / 1$ - 3 times, the patients' 6 months and 1 year survival rates were $74.0 \%$ and $47.0 \%$, median survival time was 56 weeks, local tumor control rate was $83.0 \%$. Our results show that 1 week after treatment by the CyberKnife, the clinical symptoms improve the effective rate was $95.2 \%$; 3 months After CyberKnife treatment and effectiveness were $82.5 \%$ and $96.8 \%$ respectively; the patient's survival rate was $82.5 \%$ at 6 months; 1 year survival rate was 55.6\%; the median survival time was 16 months. CyberKnife is a new technology; our follow-up time is short; long-term efficacy and late tissue damage still need to explore and 
study.

It is difficult to determine the nature of the lesions without obvious clinical symptoms and MRI showed progression [7] [8]. Asao et al. [9] reported radioactive damage area increased ADC values, and tumor recurrence area $\mathrm{ADC}$ values is not increased, the ADC values of tumor recurrence are markedly lower than the radioactive damage area. MRI diffusion-weighted imaging can help assess the efficacy of brain metastases in patients. The results indicate that in 52 patients (67 lesions) review ADC values after treatment was increased to varying degrees, the inactivation but the extent of the ADC values of quantitative analysis remains to be further research.

\section{Conclusion}

CyberKnife is an effective method for the treatment of brain metastases; MRI enhances scan and ADC values measurements help assess the efficacy of brain metastases, which is worthy of further promotion.

\section{References}

[1] Therasse, P., Arbuck, S.G., Eisenhauer, E.A., et al. (2000) New Guidelines to Evaluate the Response to Treatment in Solid Tumors. Journal of the National Cancer Institute, 92, 205-216. http://dx.doi.org/10.1093/jnci/92.3.205

[2] Yu, Z., Yin, W.B., Xu, G.Z., et al. (2008) Radiation Therapy Oncology. 4th Edition, Peking Union Medical College Press, Beijing, 796-798.

[3] Mori, Y., Kondziolka, D., Flickinger, J.C., Logan, T. and Lunsford, L.D. (1998) Stereotactic Radiosurgery for Brain Metastasis from Renal Cell Carcinoma. Cancer, 83, 344-353. http://dx.doi.org/10.1002/(SICI)1097-0142(19980715)83:2<344::AID-CNCR19>3.0.CO;2-T

[4] Mori, Y., Kondziolka, D., Flickinger, J.C., Kirkwood, J.M., Agarwala, S. and Lunsford, L.D. (1998) Stereotactic Radiosurgery for Cerebral Metastatic Melanoma: Factors Affecting Local Disease Control and Survival. International Journal of Radiation Oncology, Biology, Physics, 42, 581-589. http://dx.doi.org/10.1016/S0360-3016(98)00272-7

[5] Young, M.M., Medbery, C.A., Morrison, A.E., et al. (2005) Stereotactic Radiosurgery for Brain Metastases from Non-Small Cell Lung Cancer: Comparison of Gamma Knife and CyberKnife. Robotic Radiosurgery, 1, 97-107.

[6] Nishizaki, T., Saito, K., Jimi, Y., Harada, N., Kajiwara, K., Nomura, S., Ishihara, H., Yoshikawa, K., Yoneda, H., Suzuki, M. and Gibbs, I.C. (2006) The Role of CyberKnife Radiosurgery/Radiotherapy for Brain Metastases of Multiple or Large-Size Tumors. Minimally Invasive Neurosurgery, 49, 203-209. http://dx.doi.org/10.1055/s-2006-947998

[7] Simpson, J.R., Mendenhall, W.M., Schupak, K.D., American College of Radiology, et al. (2000) Follow-Up and Retreatment of Brain Metastasis (ACR Appropriateness Criteria). Radiology, 215, 1129-1135.

[8] Chernov, M.F., Ono, Y., Abe, K., et al. (2013) Differentiation of Tumor Progression and Radiation-Induced Effects after Intracranial Radiosurgery. In: Hayashi, M., Ganz, J.C., Takakura, K. and Chernov, M.F., Eds., Gamma Knife Neurosurgery in the Management of Intracranial Disorders, Springer, New York, 193-210.

[9] Asao, C., Korogi, Y., Kitajima, M., Hirai, T., Baba, Y., Makino, K., Kochi, M., Morishita, S. and Yamashita, Y. (2005) Diffusion-Weighted Imaging of Radiation-Induced Brain Injury for Differentiation from Tumor Recurrence. American Journal of Neuroradiology, 26, 1455-1460. 